\title{
Risk Factors for Resistance and Mortality in Patients with Extensively Resistant Acinetobacter Bacteremia in Taleghani Hospital in Tehran,
}

\section{Iran}

\author{
Soheila Abolghasemi, ${ }^{1}$ Zahra Madadi, ${ }^{2}$ and Masoud Mardani ${ }^{3,}{ }^{*}$ \\ ${ }^{1}$ Department of Infectious Diseases, Tehran Medical Sciences Branch, Islamic Azad University, Tehran, Iran \\ ${ }^{2}$ Non-Communicable Diseases Research Center, Endocrinology and Metabolism Population Sciences Institute, Tehran University of Medical Sciences, Tehran, Iran \\ ${ }^{3}$ Infectious Diseases and Tropical Medicine Research Center, Shahid Beheshti University of Medical Sciences, Tehran, Iran \\ "Corresponding author: Masoud Mardani, Infectious Diseases and Tropical Medicine Research Center, Shahid Beheshti University of Medical Sciences, Tehran, Iran. Tel: \\ +98-9121132678, E-mail: drmasoudmardani@yahoo.com
}

Received 2017 April 27; Revised 2018 January 24; Accepted 2018 January 29.

\begin{abstract}
Objectives: This study describes the antibiotic resistance pattern in patients with Acinetobacter bacteremia and investigates the factors leading to extensively drug resistance (XDR) and mortality.

Methods: This descriptive analytical study was conducted in patients with Acinetobacter bacteremia between February 2014 and February 2015. The microorganisms were identified with conventional bacteriological methods and then, following the clinical and laboratory standards institute (CLSI) recommendations, susceptibility testing was performed on all isolates. Moreover, we used electronic records to extract the patients' data and analyzed them using Chi-square test or Fisher's exact test and T-Test.

Results: Among the influencing factors, the severity of clinical symptoms at the time of admission, longer duration of hospitalization especially in the ICU, and use of invasive devices such as ventilator had a significant relationship with the occurrence of resistance and mortality in the patients. In addition, the central venous catheterization and history of drug resistance such as XDR were associated with mortality.

Conclusions: Given the increasing prevalence of XDR Acinetobacter strains causing resistant nosocomial infection, especially in immunocompromised patients, it is necessary to reduce the risk factors leading to drug resistance and mortality. Furthermore, early administration of empiric antibiotic therapy can have a good effect on the outcomes.
\end{abstract}

Keywords: Bacteremia, Extensively Drug Resistance, Acinetobacter, A. Baumannii

\section{Background}

Acinetobacter is a non-fermentative gram-negative bacilli (NFGNB) with a negative oxidase reaction, which is non-motile, aerobic, and widespread in nature for instance in water, soil, vegetables, and even in skin flora (1, 2).

A. baumannii is the most common strain of this bacilli and the outbreak of the infection caused by strains resistant to multiple antibiotic classes, including carbapenems, is a serious concern in many hospitals $(1,2)$. Other strains like A. Johnsonii and A.haemolyticusare rarely have been isolated. Bacteremia due to A. lwoffii is mainly associated with vascular catheters; it also causes infections in immunocompromised patients and usually has a good prognosis $(1,2)$.

The prevalence of antimicrobial resistance among Acinetobacter species (spp.) has substantially increased over the last decade all over the world $(2,3)$. During the years 2008 - 2011, 100\% of isolated strains in Mex- ico (4) and $75.52 \%$ of all detected Acinetobacter strains in China (5) were Multidrug-Resistant (MDR). As reported by a meta-analysis conducted in Iran, the prevalence of MDR Acinetobacter increased from 50\% between the years 2001 - 2007 to $74 \%$ between the years 2010 - 2015 (6). Extensively drug-resistant (XDR) Acinetobacter, including carbapenems-producing strains, is becoming a significant public health threat as there are fewer treatment options for them $(3,7)$.

Unfortunately, colistin resistant Acinetobacter or pan drug resistant (PDR) strains are also emerging in the world (4). In Iran, the reported prevalence of this type of strains varies from zero to $12 \%(4,8)$.

The serious complications of Acinetobacter spp. such as bacteremia and multiple organ failures are prone to occur in severely immunocompromised patients $(2,3,5)$. Several studies have reported the high prevalence of carbapenemresistant strains in these patients $(3,9,10)$. It is also emphasized that some factors including admission to intensive care unit(ICU), mechanical ventilation, two or more organ 
failures, and use of invasive devices in these patients are more effective than the underlying diseases are in increasing the prevalence of drug resistance and mortality $(5,11)$.

Given the importance of the subject, this study aimed to determine the resistance pattern of this microorganism and identify the factors leading to drug resistance and mortality.

\section{Objectives}

The aim of the present study was to identify the antibacterial resistance patterns and factors leading to XDR and mortality in patients with Acinetobacter bloodstream infection admitted to Taleghani hospital in Iran within a period of about one year.

\section{Methods}

This descriptive-analytic study was conducted in Taleghani hospital in Tehran, Iran. As the expected prevalence of Acinetobacter bacteremia as a nosocomial infection was reported about $1 \%-3 \%$ in major studies (1, 2 ), we collected data through a cross-sectional study conducted from February 2014 to February 2015. According to the study inclusion criteria, of all patients who referred to the hospital, those who were hospitalized and had at least a positive blood culture for Acinetobacter spp. during the hospitalization and had signs and symptoms of infection were enrolled in the study. Patients who were not hospitalized, or those who had positive results other than blood culture, and those whose data were not accessible met the exclusion criteria.

\subsection{Microbiological Methods}

After the transmission of blood samples to the laboratory, blood and eosin-methylene blue (EMB) agar media were used and the samples were incubated at $37^{\circ} \mathrm{C}$ for 24 hours. The biochemical tests were carried out to identify different species. Catalase, oxidase, and ALK/ALK reaction were tested on triple sugar iron(TSI). Moreover, we used oxidation/fermentation (OF) test for differentiation between A. baumannii and A. lwoffii colonies.

In order to assess antimicrobial susceptibility, a suspension of each colony with half McFarland turbidity was collected, and then cultivated on Mueller Hinton agar medium. After antibiotic disks were placed on the medium and incubated at $35^{\circ} \mathrm{C}$ for 24 hours, the zone of inhibition was measured as recommended by CLSI (2013) tables (12).

In keeping with CLSI, we selected 12 antibiotics from seven different categories including Ceftriaxone (30 $\mu \mathrm{g})$, Cefotaxime (30 $\mu \mathrm{g})$, Ceftazidime (30 $\mu \mathrm{g})$, Cefepime (30 $\mu \mathrm{g})$,
Gentamycin $(10 \mu \mathrm{g})$, Amikacin (30 $\mu \mathrm{g})$, Ciprofloxacin (5 $\mu \mathrm{g})$, Imipenem $(10 \mu \mathrm{g})$, Meropenem $(10 \mu \mathrm{g})$, PiperacillinTazobactam (100/10 $\mu \mathrm{g})$, Amoxicillin-clavulanate $(30 \mu \mathrm{g})$, and Trimethoprim-Sulfamethoxazole $(25 \mu \mathrm{g})(4)$. The disks were purchased from PadtanTeb, Iran. Pseudomonas aeruginosa ATCC 27853 and E. Coli ATCC 25922 were used for quality control.

In our study, we did not use complementary methods to determine sensitivity to colistin and tigecycline. Therefore, we did not report the verified cases of PDR.

\subsection{Data Collection}

In order to investigate the factors affecting the development of resistance and mortality in patients, we used electronic records to extract patients data including the following: age, sex, hospital department, underlying disease, comorbidities, severity of clinical signs and symptoms on admission, duration of hospital stay before the development of bacteremia, use of invasive devices, history of dialysis, taking corticosteroids and other Immunosuppressors and chemotherapy during the past two weeks.

\subsection{Definition}

In this study, bacteremia was defined as a pathogen isolated from at least one blood sample in patients with clinical symptoms and signs of infection (5). Considering Pitt Bacteraemia score (PBS), the severity of the disease fell into three categories of low, moderate, and high severity. It had a grading system that was used to assess the severity of illnesses, calculated based on five items: Temperature (35.1 $-36^{\circ} \mathrm{C}$ or $39-39.9^{\circ} \mathrm{C}: 1$ point, $\leq 35^{\circ} \mathrm{C}$ or $\geq 40^{\circ} \mathrm{C}: 2$ points), blood pressure (hypotension: 2 points), mental status (disorientation: 1 point, stupor: 2 points, coma: 4 points), respiratory status (mechanical ventilation: 2 points), and cardiac status (cardiac arrest: 4 points). A sum of scores equal to 0 - 1 represented low severity, 2 - 3 represented moderate severity, and $\geq 4$ represented high severity $(5,13)$.

\subsection{Statistical Analysis}

For data description, we used two dimensions (Tables 2 and 3). The collected data were analyzed using Chisquare test or Fisher's exact test; in addition, to compare the means, we used independent samples test(T-Test). Multivariate significant factors for XDR acquisition and mortality were presented as odds ratio (OR) with a 95\% confidence interval (95\% CI).

All the tests of significance were two-sided and the results with a P value $<0.05$ were reported as significant. 


\begin{tabular}{lccc}
\hline Table 1. The Pattern of Drug Resistance in A. Baumannii Isolates & \\
\hline Antibiotics & Susceptible, \% & Intermediate, $\%$ & Resistant, \% \\
\hline Ceftriaxone & 3 & 4 & 93 \\
\hline Ceftizoxime & 3 & 4 & 93 \\
\hline Cefepime & 3 & 0 & 97 \\
\hline Ceftazidime & 23 & 4 & 73 \\
\hline Gentamycin & 17 & 3 & 80 \\
\hline Amikacin & 10 & 3 & 87 \\
\hline Ciprofloxacin & 47 & 0 & 53 \\
\hline $\begin{array}{l}\text { Imipenem } \\
\text { Meropenem }\end{array}$ & 44 & 3 & 53 \\
\hline $\begin{array}{l}\text { Pipracillin- } \\
\text { tazobactam }\end{array}$ & 20 & 0 & 80 \\
\hline $\begin{array}{l}\text { Trimetoprim- } \\
\text { sulfamethoxazole }\end{array}$ & 30 & 7 & 69 \\
\hline \begin{tabular}{l} 
Amoxi-clavunate \\
\hline
\end{tabular} & 3 & 3 & 97 \\
\hline
\end{tabular}

\section{Results}

Of a total of 3436 blood culture samples sent to the laboratory of Taleghani hospital, 276 positive cultures were isolated. Of these, 210 samples were gram-negative bacteria. Among the isolated gram-negative strains, 90 samples were NFGNB that included 57 Pseudomonas, 32 Acinetobacter, and one Stenotrophomonasmaltophilia.

Since we detected positive Acinetobacter blood cultures in 32 inpatients, the prevalence of bacteremia caused by this microorganism was estimated to be about 9.3 per thousand people (1\%). Observing the exclusion criteria, two patients were eliminated from the study and finally, we evaluated a total of 30 patients. Of the 30 selected patients, 22 patients had underlying diseases causing immunodeficiencies such as hematologic malignancies, solid tumors, history of bone marrow transplantation, organ transplantation, and renal failure. However, the other eight patients had no detectable underlying disease leading to immunodeficiency problems. Although $73 \%$ of bloodstream infections caused by Acinetobacter were observed in immunocompromised cases, as compared with other patients, there was no significant relationship between the type of underlying diseases and XDR strains (Table 2).

As shown in Table1, we checked the resistance of Acinetobacter species to 12 types of routinely reported antibiotics, and based on the definitions, we divided them into XDR and non-XDR strains.

At first, we evaluated risk factors that made patients prone to XDR Acinetobacter bacteremia. Based on the results of multivariate analysis, a hospital stay of more than 48 hours, high PBS, admission to the ICU, and use of ventila- tor were independently associated with XDR Acinetobacter bacteremia (Table 2 ).

In the next stage, we assessed the risk factors for 30-day mortality in patients with Acinetobacter bacteremia.

The mortality rate was significantly higher in XDR Acinetobacter bacteremia group than in non-XDR Acinetobacter bacteremia group and had a significant relationship with the administration of inappropriate antibiotics (not consistent with the results of antibiogram) in XDR patients (Table 3).

Furthermore, based on the results of multivariate analysis, a hospital stay of more than 14 days, high PBS, admission to ICU, use of a ventilator, and central venous catheterization were identified as the independent risk factors for 30-day mortality in patients (Table 3).

\section{Discussion}

Infection with NFGNBs such as Acinetobacter is of great importance and is considered as a major cause of healthcare-associated infections, especially among patients with immunodeficiency problems (3).

In addition to previously mentioned studies (1, 2), some research in Iran and China showed that the prevalence of Acinetobacter, as a nosocomial bloodstream infection, was about $1 \%-3 \%(5,14,15)$, that is almost similar to the results of our study which estimated a prevalence of about $1 \%$.

Given the increasing prevalence of resistance of Acinetobacter spp. to broad-spectrum antibiotics such as carbapenems, we assessed its antimicrobial susceptibility pattern (Table 1) and based on resistance classifications, we assessed risk factors that affect the resistance and outcome.

Since in most articles the general term of MDR is used to refer to a variety of strains non-susceptible to different classes of antibiotics, which indeed includes XDR and PDR cases, more attention must be paid when interpreting the results of this study and comparing it with the results of other studies (16-18).

Based on one of the most recent reviews, MDR Acinetobacter spp. is defined as an isolate resistant to at least three classes of antimicrobial agents, including penicillins and cephalosporins (and inhibitor combinations), fluoroquinolones, and aminoglycosides. XDR Acinetobacter spp. is defined as the isolate resistant to the three classes of antibiotics described above (MDR), which must also be resistant to carbapenems $(7,16,17)$. However, we must notice that carbapenem-resistant isolates often, but not always, are XDR and resistance to a carbapenem does not ensure the resistance to other types of carbapenems $(18,19)$, as in our study, all the patients with XDR strains were resistant to imipenem and meropenem, but some cases, which 
Table 2. The Comparison of Demographic and Clinical Characteristics of Patients with XDR Acinetobacter Bacteremia and Non-XDR Acinetobacter Bacteremia, Using a Multivariate $\operatorname{Model}(\mathrm{N}=30)^{\mathrm{a}}$

\begin{tabular}{|c|c|c|c|c|c|}
\hline \multirow[t]{2}{*}{ Variables } & \multirow[t]{2}{*}{ XDR, $N=16$} & \multirow[t]{2}{*}{ Non-XDR, N = 14} & \multicolumn{3}{|c|}{ Multivariate Analysis } \\
\hline & & & OR & $95 \mathrm{Cl} \%$ & P Value \\
\hline Age, mean \pm SD & $58.06 \pm 19.69$ & $54.43 \pm 16.89$ & - & - & 0.590 \\
\hline \multicolumn{6}{|l|}{ Sex } \\
\hline Male & $9(56.2)$ & $9(64.3)$ & - & - & 0.722 \\
\hline \multicolumn{6}{|l|}{ Length of hospital stay before positive blood culture } \\
\hline$<48 \mathrm{~h}$ & $0(0)$ & $12(85.7)$ & 0.14 & $(0.04-0.51)$ & 0.001 \\
\hline 2 - 14 days & $10(62.5)$ & $2(14.3)$ & 10 & $(1.64-60.92)$ & 0.011 \\
\hline$>14$ days & $6(37.5)$ & $0(0)$ & 1.60 & $(1.09-2.34)$ & 0.019 \\
\hline \multicolumn{6}{|l|}{ Underlying disease } \\
\hline Solid tumor & $5(31.2)$ & $5(35.7)$ & - & - & $>0.999$ \\
\hline Hematologic malignancy & $2(12.5)$ & $4(28.6)$ & - & - & 0.261 \\
\hline ESRD & $2(12.5)$ & $1(7.1)$ & - & - & $>0.999$ \\
\hline Transplant patients & $4(25)$ & $1(7.1)$ & - & - & 0.336 \\
\hline $\operatorname{PBS}^{\mathrm{b}}($ severity $) \geq 4$ & $12(75)$ & $1(7.1)$ & 39 & $(3.80-399.25)$ & 0.001 \\
\hline \multicolumn{6}{|l|}{ Invasive devices } \\
\hline Ventilator & $10(62.5)$ & $1(7.1)$ & 91.67 & $(2.23-210.11)$ & 0.002 \\
\hline Urinary catheter & $7(43.7)$ & $4(28.6)$ & - & - & 0.466 \\
\hline Central vein catheter & $9(56.2)$ & $5(35.7)$ & - & - & 0.299 \\
\hline Liver stent & $2(12.5)$ & $1(7.1)$ & - & - & $>0.999$ \\
\hline \multicolumn{6}{|l|}{ Comorbidity } \\
\hline Diabetes & $6(37.5)$ & $5(35.7)$ & - & - & $>0.999$ \\
\hline Heart disease and HTN & $3(18.7)$ & $4(28.6)$ & - & - & 0.675 \\
\hline Stay in the ICU & $8(50)$ & $0(0)$ & 2 & $(1.22-3.26)$ & 0.002 \\
\hline Stay in hemato-oncology wards & $3(18.7)$ & $9(64.3)$ & 0.13 & $(0.02-0.68)$ & 0.024 \\
\hline Chemotherapy use within past 14 days & $5(31.2)$ & $7(50)$ & - & - & 0.457 \\
\hline Dialysis within past 14 days & $3(18.7)$ & $3(21.4)$ & - & - & $>0.999$ \\
\hline Corticosteroid/immunosuppressor use within past 14 days & $6(37.5)$ & $6(42.8)$ & - & - & $>0.999$ \\
\hline
\end{tabular}

were only resistant to meropenem, were not included in the XDR group (Table 1).

Finally, PDR spp. is an XDR isolate resistant to Polymyxins and Tigecycline $(7,16,17)$.

Based on our results, of a total of 30 cases that were included in our study, 25 cases were A. baumannii and five cases were $A$. Iwoffii. Of the five $A$. lwoffii cases, no XDR strain was isolated. In most other studies, in line with our study, the prevalence of A.Lwoffii has been much lower than the prevalence of $A$. baumannii; moreover, the prevalence of MDR A. Iwoffii has been low and no case of XDR has been reported $(9,20)$.
Of all A. baumannii cases in our study, $84 \%$ were resistant (MDR: 20\% and XDR: 64\%); it is consistent with the reports on the prevalence of resistant Acinetobacter strains in Imam Khomeini hospital in Ahvaz in 2013 (81.3\%) and with a meta-analysis conducted in Iran from 2007 to 2015 to determine the prevalence of imipenem resistant Acinetobacter that was $55 \%(6,21)$.

Several studies have investigated the prevalence of PDR strains in Iran and they have reported a prevalence of about $0 \%$ to $12 \%(4,8)$.

It should be noted we were not able to report PDRAcinetobacter species in our study because, during the time of 


\begin{tabular}{|c|c|c|c|c|c|}
\hline \multirow[t]{2}{*}{ Variables } & \multirow[t]{2}{*}{ Survive, $N=16$} & \multirow[t]{2}{*}{ Death, $N=14$} & \multicolumn{3}{|c|}{ Multivariate Analysis } \\
\hline & & & OR & $95 \mathrm{Cl} \%$ & P Value \\
\hline Age, mean \pm SD & $54.5 \pm 18$ & $58.5 \pm 19$ & - & - & 0.558 \\
\hline Sex & & & & & 0.722 \\
\hline Male & $9(56.2)$ & $9(64.3)$ & - & - & \\
\hline Female & $7(43.8)$ & $5(35.7)$ & & & \\
\hline \multicolumn{6}{|l|}{ Resistance } \\
\hline Non-XDR & $13(81.2)$ & $1(7)$ & 0.41 & $(0.26-0.67)$ & 0.019 \\
\hline $\mathrm{XDR}$ & $3(18.8)$ & $13(92.9)$ & 0.18 & $(0.002-0.19)$ & 0.001 \\
\hline \multicolumn{6}{|l|}{ Length of hospital stay before positive blood culture } \\
\hline$<48 \mathrm{~h}$ & $12(75)$ & 0 & 0.22 & $(0.09-0.53)$ & 0.001 \\
\hline 2 - 14 days & $4(25)$ & $8(57)$ & - & - & 0.135 \\
\hline$>14$ days & 0 & $6(43)$ & 0.33 & $(0.19-0.59)$ & 0.005 \\
\hline \multicolumn{6}{|l|}{ Underlying disease } \\
\hline Solid tumor & $5(31.2)$ & $5(35.7)$ & - & - & $>0.999$ \\
\hline Hematologic malignancy & $4(25)$ & $2(4.3)$ & - & - & 0.657 \\
\hline ESRD & $2(12.5)$ & $1(7.1)$ & - & - & $>0.999$ \\
\hline Transplant patients & $1(6.2)$ & $4(28.6)$ & - & - & 0.157 \\
\hline Pitt bacteremia score $^{b}$ (severity) $\geq 4$ & 0 & $13(92.9)$ & 0.06 & $(0.009-0.39)$ & 0.001 \\
\hline \multicolumn{6}{|l|}{ Invasive devices number } \\
\hline Ventilator & 0 & $11(78.5)$ & 0.16 & $(0.06-0.44)$ & 0.001 \\
\hline Urinary catheter & $3(18.8)$ & $8(57)$ & - & - & 0.057 \\
\hline Central vein catheter & $4(25)$ & $10(71)$ & 0.13 & $(0.026-0.67)$ & 0.026 \\
\hline Liver stent & $3(18.8)$ & $3(21.5)$ & - & - & 0.228 \\
\hline \multicolumn{6}{|l|}{ Comorbidity } \\
\hline Diabetes & $7(43.8)$ & $4(28.6)$ & - & - & 0.488 \\
\hline Heart disease and HTN & $5(31.2)$ & $2(14.3)$ & - & - & 0.399 \\
\hline Stay in the ICU & 0 & $8(57.1)$ & 0.27 & $(0.14-0.54)$ & 0.001 \\
\hline Stay in hemato-oncology wards & $8(50)$ & $4(28.6)$ & - & - & 0.284 \\
\hline Chemotherapy use within past 14 days & $6(37.5)$ & $6(43)$ & - & - & $>0.999$ \\
\hline Dialysis within past 14 days & $3(18.8)$ & $3(21.5)$ & - & - & $>0.999$ \\
\hline Corticosteroid / Immunosuppressor use within past 14 days & $5(31.2)$ & $7(50)$ & - & - & 0.457 \\
\hline \multicolumn{6}{|l|}{ Antibiotic inappropriate with antibiogaram } \\
\hline XDR patients & $3(18.8)$ & $11(78.5)$ & 4.66 & $(1.71-12.72)$ & 0.005 \\
\hline Non-XDR patients & $5(31.2)$ & 0 & 0.214 & $(0.079-0.584)$ & 0.005 \\
\hline
\end{tabular}

the study, there was limited access to E-test, which is required to determine the resistance to colistin $(11,19)$.

Some studies have investigated the factors making patients vulnerable to Acinetobacter bloodstream infections
$(3,22)$. In our study, in line with several other studies $(5,11)$, there was no significant relationship between underlying diseases leading to immunodeficiency and the emergence of resistance and mortality (Tables 2,3). 
In some articles published in 2014 , it has been stated that cancer patients, (especially those with hematologic malignancies), solid organ transplant and hematologic stem cell transplant recipients are at risk of developing resistant gram-negative bacteria, especially extendedspectrum beta-lactamases (ESBL) and carbapenemresistant enterobacteriaceae (CRE). However, as reported, the risk of developing resistant Acinetobacter is more associated with hospitalization and the length of stay in the hospital and the ICU, rather than underlying diseases $(3,11,22)$.

In our study, detecting bacteremia 48 hours after admission had a direct relationship with the emergence of resistant strains (Table 2 ) and detecting the bacteremia after more than two weeks had a relationship with increased mortality (Table 3 ). It is consistent with the results of the majority in other studies (3, 5, 23-25).

The definition of hospital-acquired bloodstream infection, i.e. an infection emerging 48 hours after hospital admission $(5,24,25)$, clarifies the impact of healthcareassociated infections in the acquisition of resistant types of this organism. In addition, increased mortality in patients with a long stay in a hospital highlights the effect of debilitating factors in hospital on patients.

Furthermore, as found in this study, admission to the ICU had a significant effect on the development of XDR Acinetobacter bacteremia and mortality. These results are in line with the results of other studies $(3,5,16,24)$. For example, in a study in India that was conducted on infants during 2010 -2012, long stay in the pediatric intensive care unit (PICU) was identified as a major factor predisposing to XDR Acinetobacter infections (24)

In some studies, the increase in PBS is considered as one of the factors increasing the risk of resistance and mortality in patients with Acinetobacter bacteremia, especially in patients with hematologic malignancy $(5,9)$. In our study, similar to the mentioned studies, $75 \%$ of patients with XDR Acinetobacter bacteremia and 93\% of mortalities in infected patients were associated with severe symptoms on admission, as measured based on PBS.

In addition, like some other reports, in the present study, we did not find a significant relationship between the history of using corticosteroid and non-corticosteroid immunosuppressors, chemotherapy, and the emergence of resistance and mortality (5). Indeed similar to other studies, these factors in immunocompromised patients make them prone to infections caused by Acinetobacter spp.; but other factors are more important in developing resistance $(3,5,9,22)$.

According to several studies, renal failure with or without dialysis is a risk factor for developing multidrug resistance $(2,23)$. In our study, as only five patients were under- going dialysis, we did not find any relationship between dialysis and the emergence of resistance and mortality.

To our knowledge, every invasive procedure in hospitalized patients, especially in those admitted to the ICU, can accelerate the process of infection. Among the invasive devices used during hospitalization, especially for patients admitted to ICU, mechanical ventilation had a significant relationship with developing resistant strains and mortality (Tables 1, 2). Moreover, the use of central venous catheterization was associated with poor prognosis (Table $3)$. These results are consistent with the results of other studies $(3,16,24)$.

Several studies have been conducted to investigate the use of antibiotic therapy before performing culture test and antibiogram in nosocomial infections, especially caused by Acinetobacter; as most of them have reported, the inappropriate empiric antibiotic administration can be a major reason for bad prognosis in severe infections $(5,18)$. In our study, inappropriate antimicrobial therapy was significantly more associated with poor outcome and high mortality in XDR patients than in non-XDRs. Because of the delay in starting the administration of appropriate antibiotics in most patients, all the 16 XDR patients were included in "inappropriate antimicrobial groups"; so, we were not able to show differences between treatment groups in terms of receiving appropriate or inappropriate antimicrobial therapy.

Previous studies have recommended the use of combination therapy including colistin, which must be administered based on the severity of the disease and epidemiological results, especially in an area with a high prevalence of XDR or carbapenem-resistant strains, even before obtaining the results of susceptibility tests; according to the mentioned studies, it could be much better than waiting for the results of microbiological tests as it could reduce mortality $(1,18)$.

\subsection{Conclusion}

Based on the results of studies conducted in Iran and other countries, the prevalence of XDR Acinetobacter strains is apparently rising although there are a very limited number of reported cases of PDR. To reduce the prevalence of the disease and mortality especially in immunocompromised patients, it is recommended to adopt preventive measures such as early discharge of patients from the ICU, removing unnecessary invasive devices, early onset of empiric antibiotics in patients with severe symptoms on the basis of resistance patterns in the region or in the treatment center, and through combination therapy using colistin and carbapenems, sulbactam or rifampin.

Because of the probable increase in the prevalence of PDR strains in our country, in addition to recognizing the 
risk factors predisposing people to infection, it is recommended to improve and revise antibiotic prescription and infection control policies in future.

\section{Acknowledgments}

The authors would like to express their thanks to the staff working in the clinical research development center of Bou-Ali hospital, Tehran.

\section{Footnotes}

Authors' Contribution: Study concept and design: Dr. Masoud Mardani; collection of data, analysis, and interpretation of data: Dr. Soheila Abolghasemi; drafting of the manuscript: Dr. Soheila Abolghasemi, Miss. Zahra Madadi; statistical analysis, administrative, technical, and material support: Dr. Soheila Abolghasemi; study supervision: Dr. Masoud Mardani.

Conflict of Interest: The authors do not have any conflict of interest.

\section{References}

1. Kasper D, Jameson JL, Hauser S, Loscalzo J, Fauci A, Longo D. Harrison's Principles of Internal Medicine 19/E. 1 \&amp; 2. McGraw-Hill Education; 2015. p. 1036-8.

2. Bennett JE, Dolin R, Blaser MJ. Mandell, Douglas, and Bennett's Principles and Practice of Infectious Diseases. Elsevier Health Sciences; 2014.

3. Al-Anazi KA, Al-Jasser AM. Infections Caused by Acinetobacter baumannii in Recipients of Hematopoietic Stem Cell Transplantation. Front Oncol. 2014;4:186. doi: 10.3389/fonc.2014.00186. [PubMed: 25072028]. [PubMed Central: PMC4095644].

4. Vakili B, Fazeli H, Shoaei P, Yaran M, Ataei B, Khorvash F, et al. Detection of colistin sensitivity in clinical isolates of Acinetobacter baumannii in Iran. J Res Med Sci. 2014;19(Suppl 1):S67-70. [PubMed: 25002899]. [PubMed Central: PMC4078383].

5. Liu Q, Li W, Du X, Li W, Zhong T, Tang Y, et al. Risk and Prognostic Factors for Multidrug-Resistant Acinetobacter Baumannii Complex Bacteremia: A Retrospective Study in a Tertiary Hospital of West China. PLoS One. 2015;10(6). e0130701. doi: 10.1371/journal.pone.0130701. [PubMed: 26083415]. [PubMed Central: PMC4471170].

6. Pourhajibagher M, Hashemi FB, Pourakbari B, Aziemzadeh M, Bahador A. Antimicrobial Resistance of Acinetobacter baumannii to Imipenem in Iran: A Systematic Review and Meta-Analysis. Open Microbiol J. 2016;10:32-42. doi: 10.2174/1874285801610010032. [PubMed: 27099638]. [PubMed Central: PMC4814728].

7. Dedeic-Ljubovic A, Granov D, Hukic M. Emergence of extensive drugresistant (XDR) Acinetobacter baumanniiin the Clinical Center University of Sarajevo, Bosnia and Herzegovina. Med Glas (Zenica). 2015;12(2):169-76. doi: 10.17392/809-15. [PubMed: 26276655].

8. Yadegarynia D, Azad MK, Gachkar L, Roodsari SR, Arab-Mazar Z. Drug resistance of acinetobacter in selected hospitals. Novelty Biomed. 2015;3(3):103-10.

9. Metan G, Demiraslan H, Kaynar LG, Zararsiz G, Alp E, Eser B. Factors influencing the early mortality in haematological malignancy patients with nosocomial Gram negative bacilli bacteraemia: a retrospective analysis of 154 cases. Braz J Infect Dis. 2013;17(2):143-9. doi: 10.1016/j.bjid.2012.09.010. [PubMed: 23485438].
10. El-Mahallawy HA, Hamid RMA, Hassan SS, Radwan S, Saber M. The increased frequency of carbapenem resistant non fermenting gram negative pathogens as causes of health care associated infections in adult cancer patients. J Cancer Ther. 2015;6(10):881-8. doi: 10.4236/jct.2015.610096.

11. Perez F, Adachi J, Bonomo RA. Antibiotic-resistant gram-negative bacterial infections in patients with cancer. Clin Infect Dis. 2014;59 Suppl 5:S335-9. doi: 10.1093/cid/ciu612. [PubMed: 25352627]. [PubMed Central: PMC4303050].

12. CLSI . Performance Standards for Antimicrobial Susceptibility Testing; Twenty-Third Informational Supplement. Clinical \& Laboratory Standards Institute; 2013.

13. Al-Hasan MN, Juhn YJ, Bang DW, Yang HJ, Baddour LM. External validation of bloodstream infection mortality risk score in a populationbased cohort. Clin Microbiol Infect. 2014;20(9):886-91. doi: 10.1111/14690691.12607. [PubMed: 25455590]. [PubMed Central: PMC4254722].

14. Poorzargar P, Javadpour S, Karmostaji A. Distribution and antibiogram pattern of Acinetobacter infections in Shahid Mohammadi hospital, Bandar Abbas, Iran. Hormozgan Med J. 2017;20(6):422-9. doi: 10.18869/acadpub.hmj.20.6.422.

15. Mahmoudi S, Mahzari M, Banar M, Pourakbari B, Haghi Ashtiani MT, Mohammadi M, et al. Antimicrobial resistance patterns of Gramnegative bacteria isolated from bloodstream infections in an Iranian referral paediatric hospital: A 5.5-year study. J Glob Antimicrob Resist. 2017;11:17-22. doi: 10.1016/j.jgar.2017.04.013. [PubMed: 28729206].

16. Manchanda V, Sanchaita S, Singh N. Multidrug resistant acinetobacter. J Glob Infect Dis. 2010;2(3):291-304. doi: 10.4103/0974-777X.68538. [PubMed: 20927292]. [PubMed Central: PMC2946687].

17. Magiorakos AP, Srinivasan A, Carey RB, Carmeli Y, Falagas ME, Giske CG, et al. Multidrug-resistant, extensively drug-resistant and pandrug-resistant bacteria: an international expert proposal for interim standard definitions for acquired resistance. Clin Microbiol Infect. 2012;18(3):268-81. doi: 10.1111/j.1469-0691.2011.03570.x. [PubMed: 21793988].

18. Viehman JA, Nguyen MH, Doi Y. Treatment options for carbapenemresistant and extensively drug-resistant Acinetobacter baumannii infections. Drugs. 2014;74(12):1315-33. doi: 10.1007/s40265-014-0267-8. [PubMed: 25091170]. [PubMed Central: PMC4258832].

19. Sarmad ZG, Eftekhar F. Prevalence of Carbapenem resistance in acinetobacter baumannii isolates from burn and non-burn patients in Tehran. J Med Bacteriol. 2015;4(3-4):56-60.

20. Rattanaumpawan P, Ussavasodhi P, Kiratisin P, Aswapokee N. Epidemiology of bacteremia caused by uncommon non-fermentative gram-negative bacteria. BMC Infect Dis. 2013;13:167. doi: 10.1186/14712334-13-167. [PubMed: 23566113]. [PubMed Central: PMC3636083].

21. Izadpour F, Ranjbari N, Aramesh MR, Moosavian M, ShahAli S, Larki $\mathrm{F}$, et al. An Investigation of Antibacterial Resistance Patterns Among Acinetobacter baumannii and Pseudomonas aeruginosa Isolates Collected from Intensive Care Units of a UniversityAffiliated Hospital in Ahvaz, Iran. Jundishapur J Microbiol. 2016;9(8). e35624. doi: 10.5812/jjm.35624. [PubMed: 27800136]. [PubMed Central: PMC5080913].

22. Cervera C, van Delden C, Gavalda J, Welte T, Akova M, Carratala J, et al. Multidrug-resistant bacteria in solid organ transplant recipients. Clin Microbiol Infect. 2014;20 Suppl 7:49-73. doi: 10.1111/1469-0691.12687. [PubMed: 24861521].

23. van Duin D, van Delden C, AST Infectious Diseases Community of Practice . Multidrug-resistant gram-negative bacteria infections in solid organ transplantation. Am J Transplant. 2013;13 Suppl 4:31-41. doi: 10.1111/ajt.12096. [PubMed: 23464996].

24. Kapoor K, Jain S, Jajoo M, Dublish S, Dabas V, Manchanda V. Risk Factors and Predictors of Mortality in Critically ill Children with Extensively-Drug Resistant Acinetobacter baumannii Infection in a Pediatric Intensive Care Unit. Iran J Pediatr. 2014;24(5):569-74. [PubMed: 25793063]. [PubMed Central: PMC4359409]. 
25. Karkhane M, Pourhoseingholi MA, Kimiia Z, Mortazavi SM, Akbariyan Torkabad MR, Hossieni Aghdam SK, et al. Attitudes toward nosocomial infections associated mortality at intensive care units, and evaluation of the risk factors. Arch Clin Infect Dis. 2016;11(2). doi: 10.5812/archcid.22504 